\title{
Long-Term Super-Resolution Imaging of Amyloid Structures Using Transient Binding of Thioflavin T
}

\author{
Kevin Spehar ${ }^{+,}$a Tianben Ding, $^{+, b}$, Yuanzi Sunc, Niraja Kedia ${ }^{a}$, Jin Lu' ${ }^{b}$, George R. Nahass ${ }^{\mathrm{a}}$, \\ Matthew D. Lew ${ }^{\mathrm{b}}$, Jan Bieschke, \\ ${ }^{a}$ Department of Biomedical Engineering, Washington University in St. Louis, St. Louis, MO, USA 63130 \\ ${ }^{b}$ Department of Electrical and Systems Engineering, Washington University in St. Louis, St. Louis, MO, USA 63130 \\ ${ }^{c}$ MRC Prion Unit, UCL Institute of Prion Diseases, Gower Street, London WC1E 6BT, UK \\ ${ }^{+}$These authors contributed equally to this work \\ mdlew@wustl.edu,j.bieschke@ucl.ac.uk
}

\begin{abstract}
Amyloids are implicated in Alzheimer's disease but cannot be well resolved by standard light microscopy. We developed a tool to directly image native amyloid structures and dynamics at nanometer resolution over minutes to days. () 2019 The Authors

OCIS codes: 170.3880 Medical and biological imaging, 100.6640 Superresolution, 170.2520 Fluorescence microscopy
\end{abstract}

Nanometer-sized aggregation intermediates are the main culprits in amyloid toxicity [1] in Alzheimer's disease (AD), but they cannot be resolved by conventional light microscopy. Single-molecule (SM) super-resolution (SR) fluorescence microscopy techniques, such as (d)STORM [2, 3], overcome the optical diffraction barrier $(\sim 250 \mathrm{~nm}$ for visible light) and visualize structures with nanoscale resolution. However, biological targets must be densely labeled with fluorescent molecules [4, 5], which can potentially alter the structure of interest. Furthermore, photobleaching of tagged fluorescent molecules limits measurement time and prevents long-term imaging of targets.

A related SM-SR technique, called PAINT [6], uses combinations of fluorophore binding and unbinding, diffusion into and out of the imaging plane, and/or spectral shifts upon binding to generate flashes of SM fluorescence. When in the immediate vicinity of their target, these probes either become fluorescent, temporarily bind to the target, or both, thereby creating a "flash" of fluorescence that is used to locate the target of interest. These blinking events are recorded over time, and image-processing algorithms [7] measure the position of each bright molecule with high precision to reconstruct a SR image.

Herein, we report a technique to image native amyloid structures using Thioflavin T (ThT) on the nanometer scale, called Transient Amyloid Binding (TAB) imaging [8]. ThT fluorescence increases upon binding to amyloid proteins, transforming dark ThT in solution into its bright state $[9,10]$. The molecules emit fluorescence until they photobleach or dissociate from the structure, enabling us to record movies of "blinking" ThT molecules, localize their positions, and reconstruct the amyloid structure (Fig. 1A, B, C).
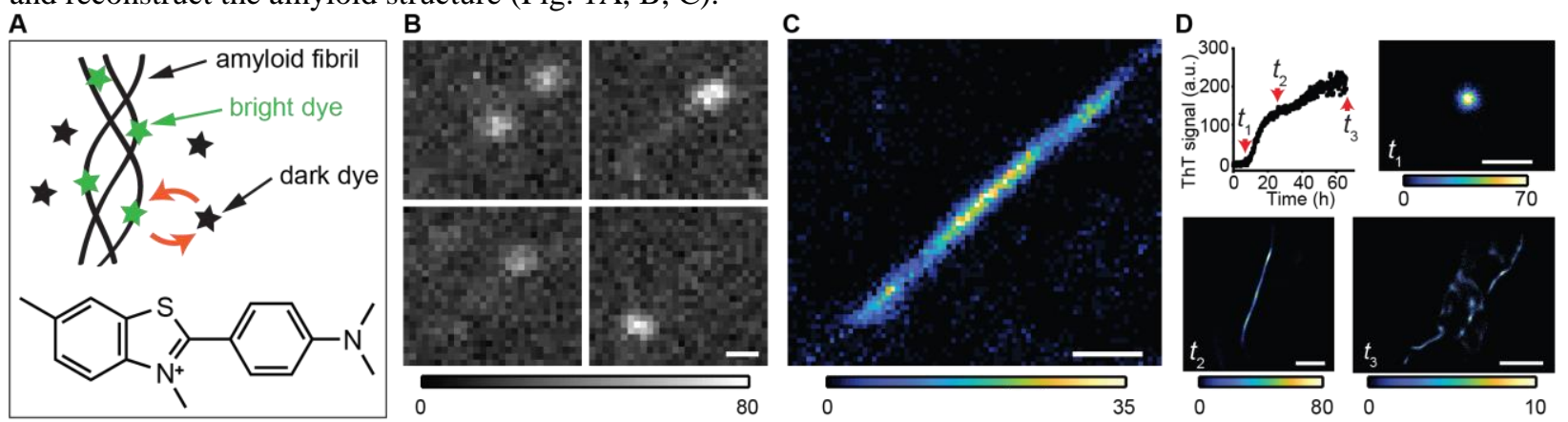

Fig 1. Demonstration of TAB imaging. (A) TAB mechanism explained: unbound dye molecules are dark while bound dyes are bright. (B) ThT blinking events on an A $\beta 42$ fibril using TAB. Scale bar: $300 \mathrm{~nm}$. Grey scale: photons per pixel. (C) A $\beta 42$ fibril SR image reconstructed from the raw data in B. Scale bar: $300 \mathrm{~nm}$. Color scale: localization per bin. (D) Top-left depicts the $A \beta 40$ aggregation kinetic denoting $\mathrm{t}_{1}(8 \mathrm{~h})$, $\mathrm{t}_{2}$ $(24 h)$, and $t_{3}(66 \mathrm{~h})$. SR images corresponding these time points show an oligomer, a fibril, and a fibril cluster. Scale bar: $0.5\left(t_{1}\right), 1\left(t_{2}\right)$, and $2.5 \mu \mathrm{m}\left(\mathrm{t}_{3}\right)$.

Accurate imaging of oligomeric structures is crucial to capturing the dynamics of $A \beta$ aggregation and could open the door for future applications in cellular imaging of oligomeric structures. To demonstrate the versatility of TAB imaging, we imaged $A \beta 42$ fibrils and $A \beta 40$ aggregates with varyious morphologies (Fig. 1C, D), including oligomers, fibrils, and fibril clusters.

To image amyloid dynamics, it is essential to have a robust tool that can follow a single aggregate over hours or more. The continuous binding and unbinding of ThT from its amyloid target enables TAB imaging to maintain a 
constant localization rate over 24 hours when imaging a single A $\beta 42$ fibril (Fig. 2A, B). Therefore, TAB imaging can produce multiple time-lapse SR images with minute-scale temporal resolution over extended periods.

We next validated the capability of TAB for dynamic SR imaging. The time-lapse images (Fig. 2C-E) show the dissolution and remodeling of A 442 fibrils by epi-gallocatechin gallate (EGCG) [11]. Remarkably, TAB imaging captured the structural dynamics of amyloid fibrils for $\sim 2$ days, thus allowing us to observe remodeling over tens of micrometers with $\sim 16 \mathrm{~nm}$ precision. In this experiment, we observed dynamics that were slower than at $37^{\circ} \mathrm{C}$ in solution [11], most likely due to the lack of agitation of fibrils that were adsorbed to the glass surface and to incubation at room temperature.
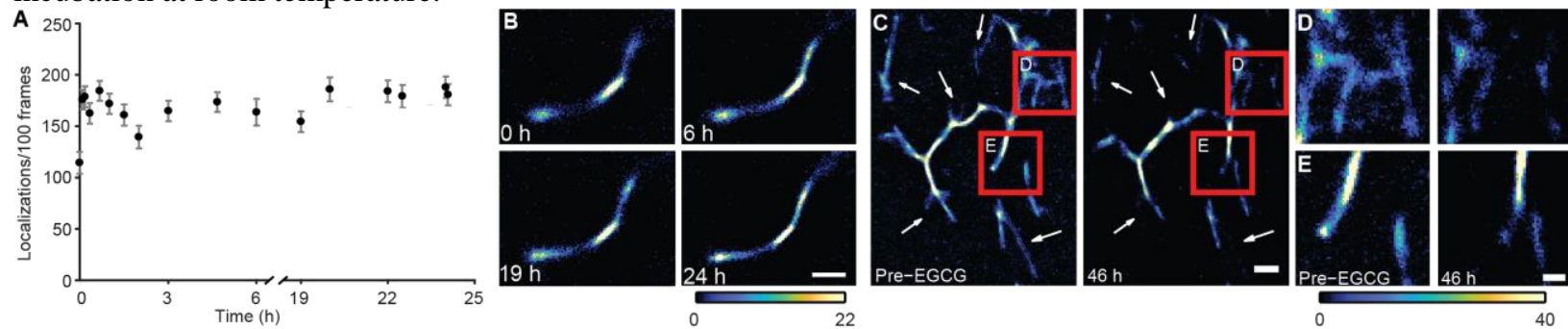

Fig 2. Long-term $\mathrm{TAB}$ imaging. (A) Average number of localizations $/ 100$ frames for each time point captured of a single A $\beta 42$ fibril. (B) SR images of the A $\beta 42$ fibril at the time points in A. Scale bar: $500 \mathrm{~nm}$. (C) A 342 fibrils before addition of EGCG and 46 hours after adding EGCG. Scale bar: $500 \mathrm{~nm}$. (D, E) Zoom-ins of portions of the structure in C showing fibril remodeling. Scale bar: $200 \mathrm{~nm}$.

Previous SR studies had limited observation times due to photobleaching and due to the permanent attachment of probes to the amyloid fibril [12]. Because ThT probes continuously dissociate and new ThT molecules rebind to amyloid, TAB imaging is inherently resistant to photobleaching. The success of these experiments demonstrates the ability of TAB imaging to follow the dynamics of amyloid structures with nanometer resolution. This capability will be essential for visualizing drugs acting on amyloid structures in order to gain insight into their molecular-scale interactions with these structures.

In summary, TAB SR microscopy is a flexible imaging technique that can provide images of amyloid structures with nanometer resolution over observation times of hours. It is capable of imaging various stages of amyloid aggregation as well as dynamic imaging of fibrillar remodeling by an anti-amyloid drug. Nanoscale imaging of aggregation intermediates will provide a clearer understanding of which structures are toxic to cells and will pave the road for further study into molecular mechanisms of AD and other amyloid diseases.

\section{References}

[1] E. Cohen, J. Bieschke, R. M. Perciavalle, J. W. Kelly, and A. Dillin, “Opposing Activities Protect Against Age-Onset Proteotoxicity," Science, vol. 313, no. 5793, pp. 1604-1610 (2006).

[2] M. J. Rust, M. Bates, and X. Zhuang, "Sub-diffraction-limit imaging by stochastic optical reconstruction microscopy (STORM)," Nature Methods, vol. 3, no. 10, pp. 793-796 (2006).

[3] M. Heilemann, S. Van De Linde, M. Schüttpelz, R. Kasper, B. Seefeldt, A. Mukherjee, P. Tinnefeld, and M. Sauer, "Subdiffraction-Resolution Fluorescence Imaging with Conventional Fluorescent Probes," Angew Chem Int Ed Engl, vol. 47, no. 33, pp. 6172-6176 (2008).

[4] H. Shroff, C. G. Galbraith, J. A. Galbraith, and E. Betzig, "Live-cell photoactivated localization microscopy of nanoscale adhesion dynamics," Nature Methods, vol. 5, no. 5, pp. 417-423 (2008).

[5] R. P. J. Nieuwenhuizen, K. A. Lidke, M. Bates, D. L. Puig, D. Grünwald, S. Stallinga, and B. Rieger, "Measuring image resolution in optical nanoscopy," Nature Methods, vol. 10, no. 6, pp. 557-562 (2013).

[6] A. Sharonov and R. M. Hochstrasser, "Wide-field subdiffraction imaging by accumulated binding of diffusing probes," Proceedings of the National Academy of Sciences, vol. 103, no. 50, pp. 18911-18916 (2006).

[7] D. Sage, H. Kirshner, T. Pengo, N. Stuurman, J. Min, S. Manley, and M. Unser, "Quantitative evaluation of software packages for singlemolecule localization microscopy," Nature Methods, vol. 12, no. 8, pp. 717-724 (2015).

[8] K. Spehar, T. Ding, Y. Sun, N. Kedia, J. Lu, G. R. Nahass, M. D. Lew, and J. Bieschke, "Super-resolution Imaging of Amyloid Structures over Extended Times by Using Transient Binding of Single Thioflavin T Molecules," ChemBioChem, vol. 19, no. 18, pp. 1944-1948 (2018).

[9] M. Biancalana and S. Koide, "Molecular mechanism of Thioflavin-T binding to amyloid fibrils," Biochimica et Biophysica Acta (BBA) Proteins and Proteomics, vol. 1804, no. 7, pp. 1405-1412 (2010).

[10] A. I. Sulatskaya, I. M. Kuznetsova, M. V. Belousov, S. A. Bondarev, G. A. Zhouravleva, and K. K. Turoverov, "Stoichiometry and Affinity of Thioflavin T Binding to Sup35p Amyloid Fibrils," Plos One, vol. 11, no. 5 (2016)

[11] J. Bieschke, J. Russ, R. P. Friedrich, D. E. Ehrnhoefer, H. Wobst, K. Neugebauer, and E. E. Wanker, "EGCG remodels mature $\alpha$-synuclein and amyloid- $\beta$ fibrils and reduces cellular toxicity," Proceedings of the National Academy of Sciences, vol. 107, no. 17, pp. 7710-7715 (2010).

[12] J. Ries, V. Udayar, A. Soragni, S. Hornemann, K. P. R. Nilsson, R. Riek, C. Hock, H. Ewers, A. A. Aguzzi, and L. Rajendran, "Superresolution Imaging of Amyloid Fibrils with Binding-Activated Probes," ACS Chemical Neuroscience, vol. 4, no. 7, pp. 1057-1061 (2013). 